\title{
CIRCULAR POLARIZATION VARIABILITY IN EXTRAGALACTIC SOURCES ON TIME SCALES OF MONTHS TO DECADES *
}

\author{
HUGH D. ALLER, MARGO F. ALLER and RICHARD M. PLOTKIN \\ University of Michigan Department of Astronomy
}

\begin{abstract}
We present previously unpublished circular polarization (cp) measurements at 4.8 and 8.0 GHz made with the University of Michigan 26-meter prime focus telescope during 1978-1984 and results of a new observational program during the past two years. Based on the preliminary analysis of our recent data, eight sources were detected at $4.8 \mathrm{GHz}$ with average degrees of cp ranging from $\leq 0.1 \%$ to $\geq 1 \%$. The results are compared with observations at other frequencies and with linear polarization and total flux density variability in the sources. The behavior of the $\mathrm{cp}$ variability observed to date is consistent with stochastic variations produced by mode conversion in transient, opaque emitting regions in the sources. The observed sign changes observed between different epochs and different frequencies are not consistent with the hypothesis that sources maintain a fixed handedness of $\mathrm{cp}$.
\end{abstract}

Keywords: circular polarization, variability

\section{Introduction}

Circular polarization (cp) measurements provide a unique insight into the physical processes in a synchrotron emitting region, even though the degrees of circular polarization are typically less than a percent in extragalactic sources (Legg and Westfold, 1968; Jones and O'Dell, 1977a; Jones and O'Dell, 1977b). Although usually weak, circular polarization has been detected in a significant number of extragalactic sources (Weiler and de Pater, 1983; Komesaroff et al., 1984; Homan and Wardle, 1999; Rayner, Norris and Sault, 2000; Homan, Attridge, and Wardle, 2001; Wardle et al., 1998) and in the galactic center (Bower, Falcke, and Backer, 1999; Bower et al., 2002; Sault and Macquart, 1999). Time variability in the circular polarization of extragalactic radio sources was detected in two extragalactic sources with the University of Michigan 26-meter telescope in the mid-1970's (Hodge and Aller, 1977). More recent measurements using the VLBA confirm that the circular polarization arises from the (presumably partially opaque) regions near the cores of relativistic jet structures and provide evidence that the emission may be be produced by an electron-positron gas (Wardle et al., 1998). We present here previously unpublished cp measurements at 4.8 and $8.0 \mathrm{GHz}$ made with the University of Michigan 26-meter prime focus telescope during 1978-1984 and new data obtained during the past two years. We now have measurements with the same

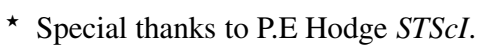


4.8 GHz INSTRUMENTAL POLARIZATION (1979)
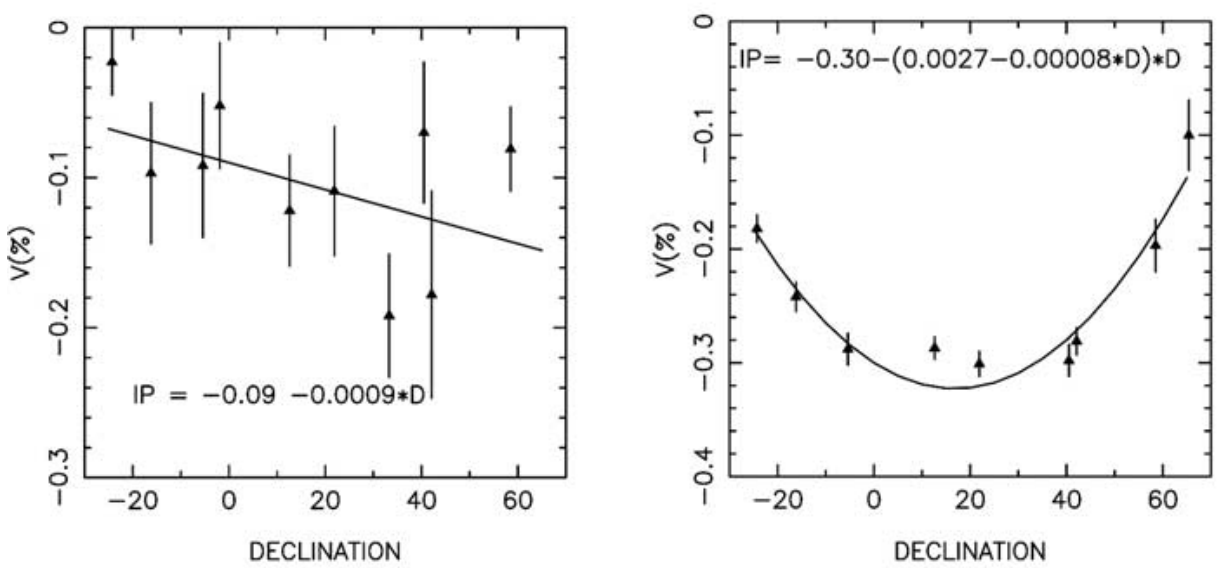

8.0 GHz INSTRUMENTAL POLARIZATION (1979)

8.0 GHz INSTRUMENTAL POLARIZATION (2002)
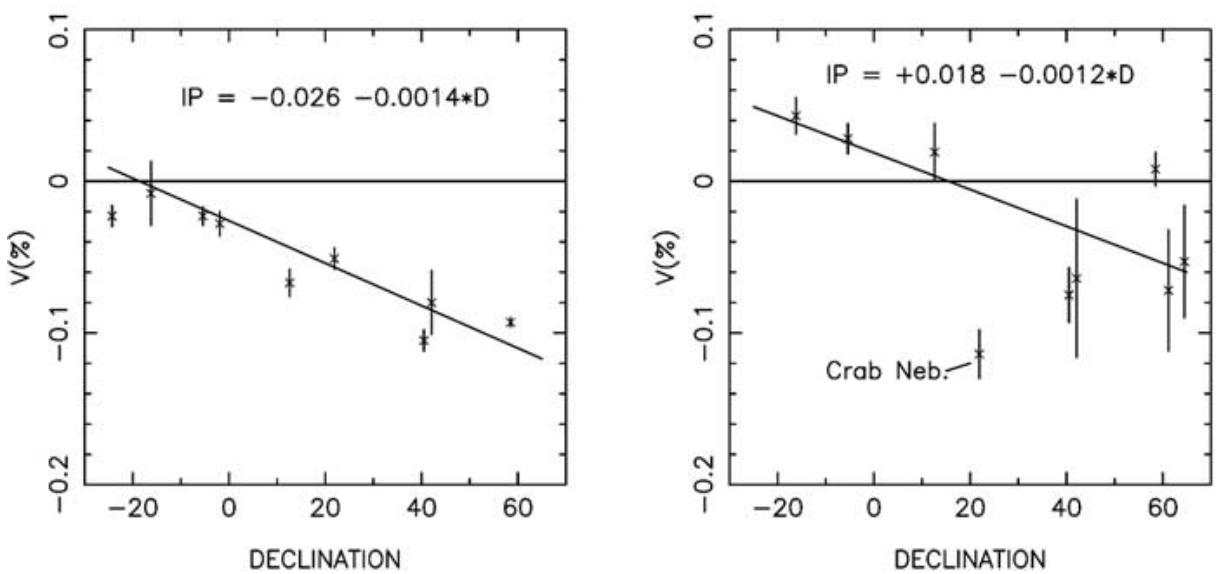

Figure 1. The instrumental polarization versus declination at 4.8 and $8.0 \mathrm{GHz}$ during the two time periods. The curves shown are empirical fits to the time-averaged data in each period.

instrument spanning more than 25 years. The sources included in this study were selected on the basis of the expectation that they might exhibit circularly polarized emission; we emphasize that this group is not a statistically complete sample.

\section{Observations and Calibration}

The Dicke-switched radiometers used in these measurements consist of broad-band amplifiers ( 0.56 and $0.72 \mathrm{GHz}$ at 4.8 and $8.0 \mathrm{GHz}$ respectively) feeding video detectors followed by audio-frequency amplifiers. The systems used for the observations were cryogenically-cooled parametric and GaAs FET amplifiers. Periodic injections of noise from current stablized noise diodes are used to monitor the gains 

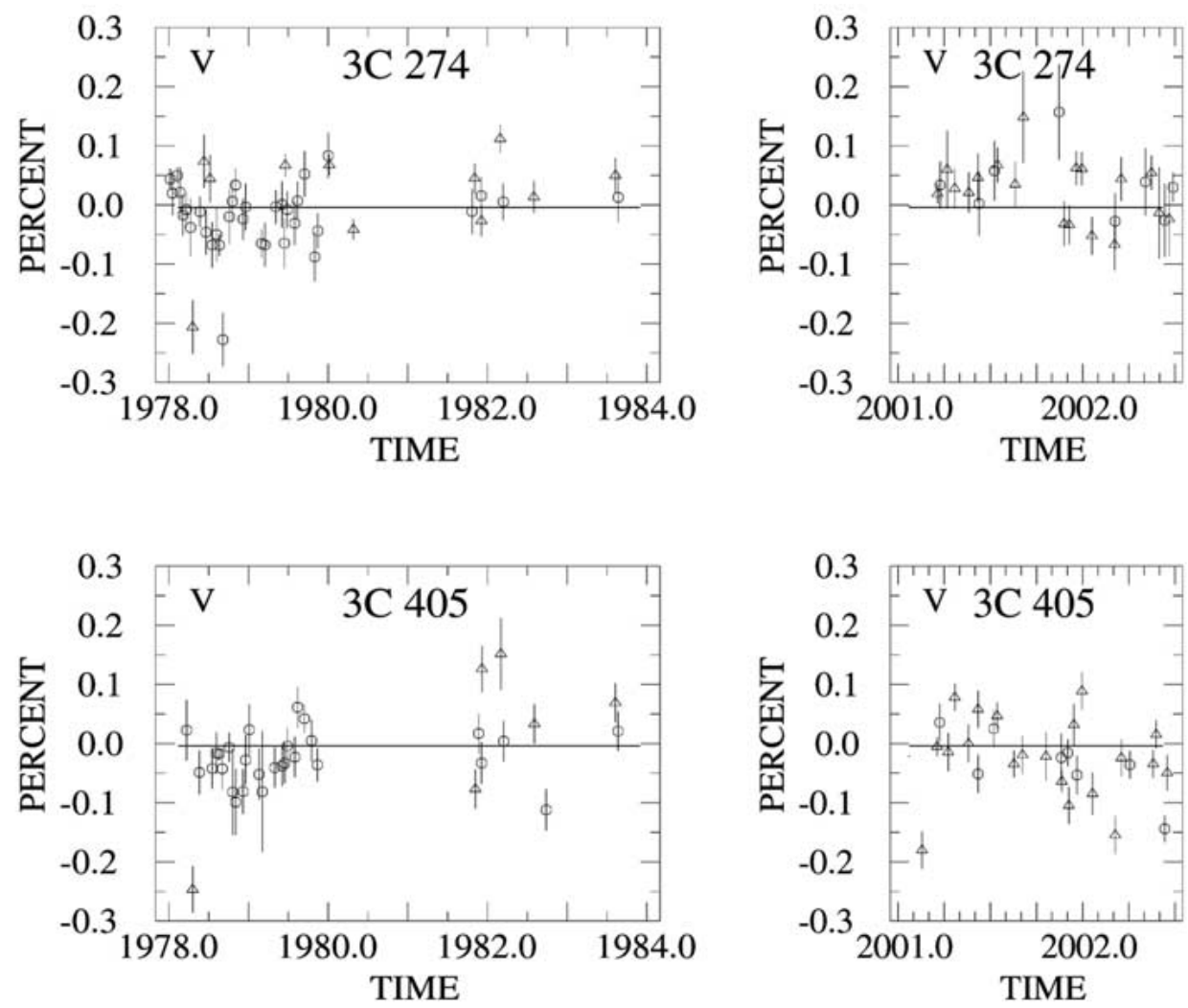

Figure 2. Two week averages of the circular polarization at 4.8 and $8.0 \mathrm{GHz}$, shown as $\Delta$ and $O$ respectively, versus time for two sources that are expected to be unpolarized. The top panels show the results for $3 \mathrm{C} 274$, and the lower panels show the results for $3 \mathrm{C} 405$ during the two time periods.

of the receiver systems The prime-focus polarimeters utilize rotating quarter-waveplates feeding orthogonal linearly-polarized transducers; the quarter-wave plates consist of tapered slabs of plastic inserted in the circular waveguide horns. The system permits the measurement of all four Stokes parameters: the total flux is determined by switching the radiometers against an off-axis feed horn, and the polarization is measured while switching between the orthogonal modes of the main horn. The absolute orientation of the wave-plates is determined from periodic observations of a linearly polarized signal source located at the vertex of the paraboloid.

The observations were made under automatic computer control. Each measurement consists of from 12 to 30150 -second off-on-off observations preceeded by position scans to verify the telescope pointing. Observations of program sources are interleaved with calibrators, approximately every two hours. Measurements were restricted to an hour angle range of less than three hours to minimize variations in instrumental effects between observations. 

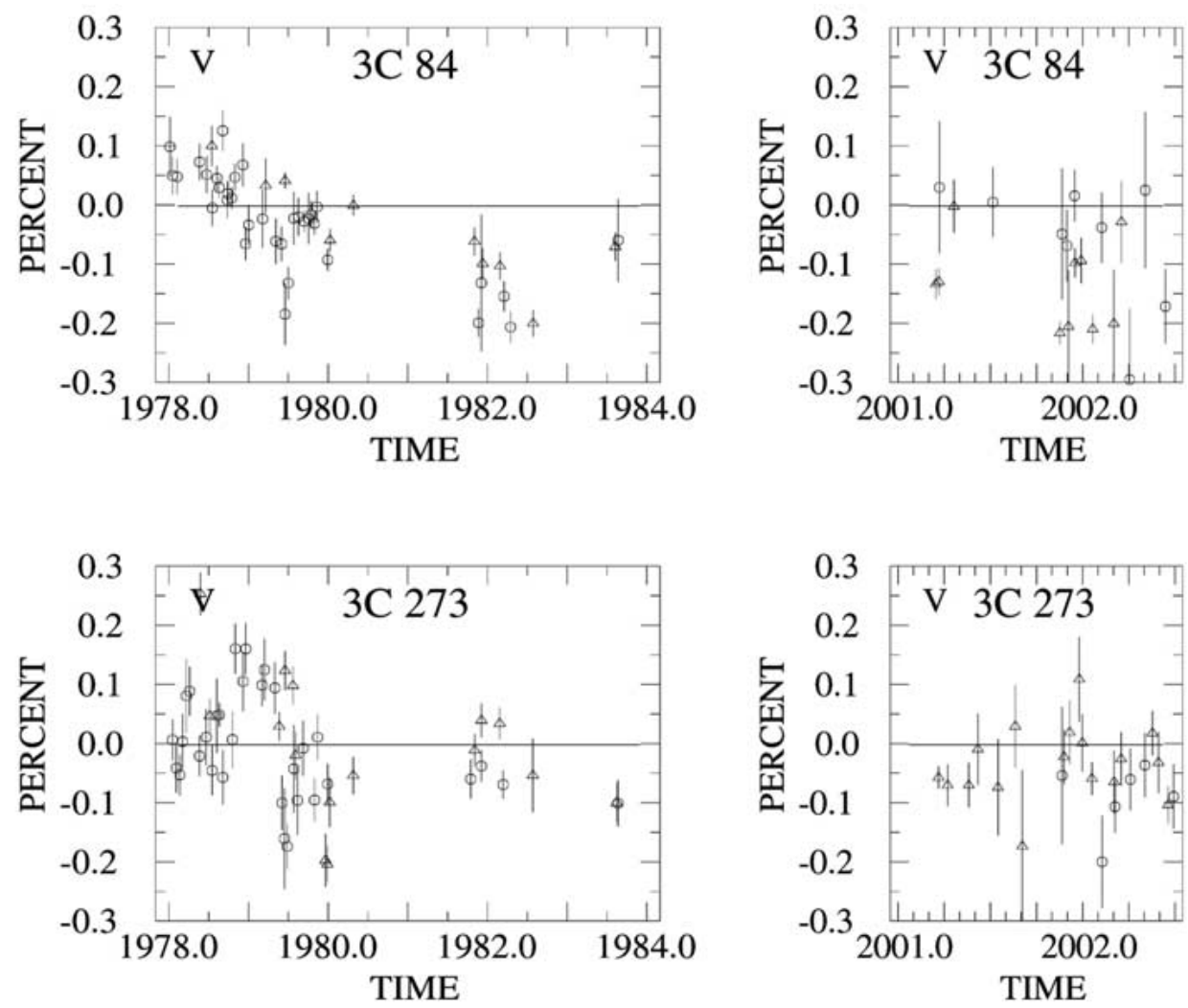

Figure 3. Two week averages of the circular polarization at 4.8 and $8.0 \mathrm{GHz}$, shown as $\Delta$ and $O$ respectively, versus time during the two time periods. The top panels show the results for $3 \mathrm{C} 84$, and the lower panels show the results for 3C 273.

The instrumental polarization is calibrated by frequent observations of the galactic HII regions M8, M17, M42 and DR21, which are assumed to be unpolarized. The time-averaged values of the cp during the two observing periods and at the two observing frequencies are shown in Figure 1. In order to better define the change in instrumental polarization with declination, these plots also include observations of two extended extragalactic objects, Virgo A and Cygnus A and several galactic supernovae remnants, 3C 58, Crab Nebula and Cassiopeia A, which we initially did not expect to be circularly polarized. The recent data at $8.0 \mathrm{GHz}$ suggests that the Crab Nebula may, in fact, be circularly polarized, and we did not include it as a calibrator; this appears to be supported by the remark made by Dr. Roger Blandford during the conference that, (based on theoretical arguments) he would expect the Crab Nebula to exhibit small amounts of cp from time to time. We interpret the observed cp values of the calibrators as the instrumental polarization introduced by the instrument. Except for the recent $4.8 \mathrm{GHz}$ results, the instrumental polarization in $\mathrm{cp}$ is of the order of $0.1 \%$, with a slow linear drift to negative Stokes parameter 

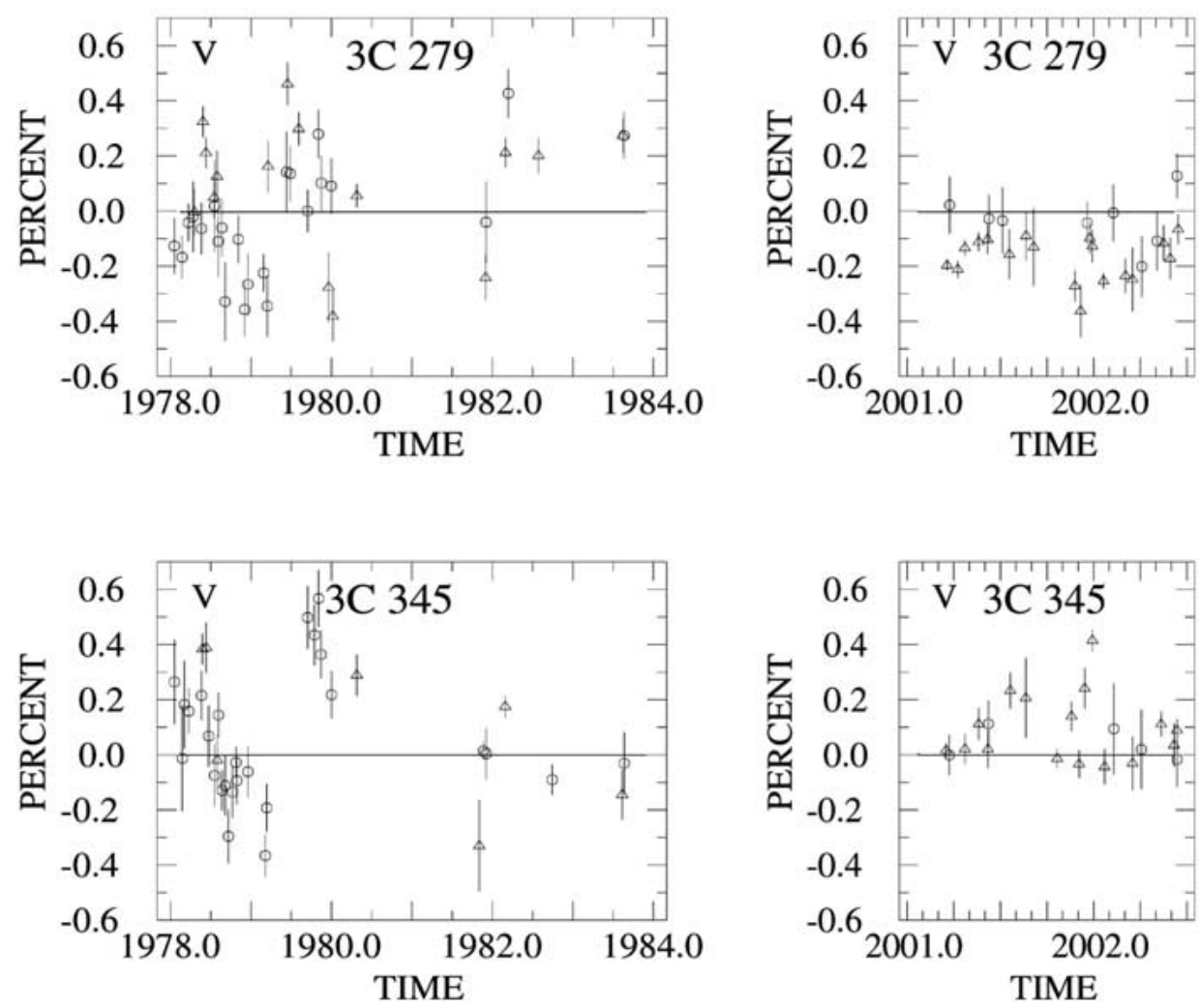

Figure 4. Two week averages of the circular polarization at 4.8 and $8.0 \mathrm{GHz}$, shown as $\Delta$ and $O$ respectively, versus time during the two time periods. The top panels show the results for $3 \mathrm{C} 279$, and the lower panels show the results for 3C 345 .

$\mathrm{V}$ with increasing declination. The instrument has proven to be remarkably stable over a 25 -year period. Shortly before initiating the recent $\mathrm{cp}$ observations we had installed a 1.2-meter diameter cone surrounding the focus area to shield the prime focus feeds from spill-over from beyond the edge of the primary reflector. Although this device has had the desirable effect of significantly lowering the system temperatures at all frequencies, it has also introduced an interaction with the horn response at $4.8 \mathrm{GHz}$. We believe that the change in the instrumental polarization with declination is due to a mechanical flexure as the antenna is moved relative to the direction of gravity; the effect is still being investigated.

There are apparent small fluctuations (of the order of $0.1 \%$ ) in the instrumental polarization between observing sessions. The correction procedure we used was to first remove the declination dependence (which does not appear to change shape with time) and then remove the average value of the instrumental polarization (the average observed $\mathrm{cp}$ of the HII regions) for each observing session. Figure 2 shows the corrected data during the two observing periods for the extended extragalactic 


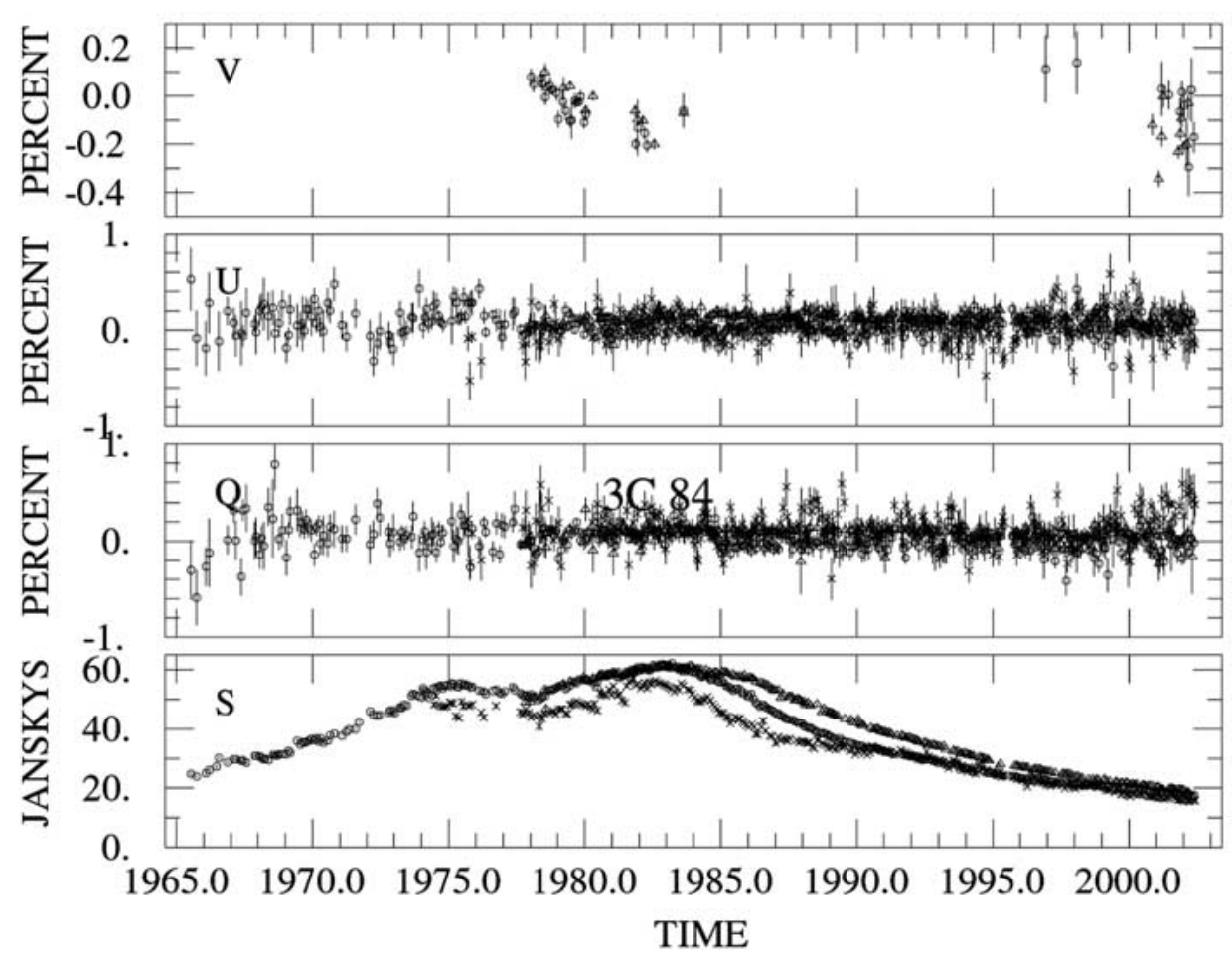

Figure 5. Monthly averages of the observed Stokes parameters for 3C 84 at 4.8, 8.0 \& $14.5 \mathrm{GHz}$, shown as $\triangle, O$, and $X$ respectively, versus time.

sources which are not expected to exhibit cp: Virgo A and Cygnus A. The data are consistent with our expectation that these sources contain no detectable cp.

\section{Observational Results}

Of the 16 extragalactic sources reported here, four, 3C 84, 3C 273, 3C 279, and 3C 345 , exhibited variability during the 1978-1984 period. The circular polarization results for these objects are shown as two-week averages in Figures 3 and 4. Figures 5 through 8 show these same sources (as monthly averages) together with the total flux density and linear polarization 26-meter data since 1965. The average circular polarizations for all 16 sources are presented in Table I: sources which exhibited variability (or suspected variability) within the time periods are designated with a $\mathbf{V}$ (or ?). Although the average cp of 3C 84 and 3C 273 during the $1978-1984$ period were not significantly different from zero, they both exhibited clear variability over the six-year period.

Below are comments on selected sources. 


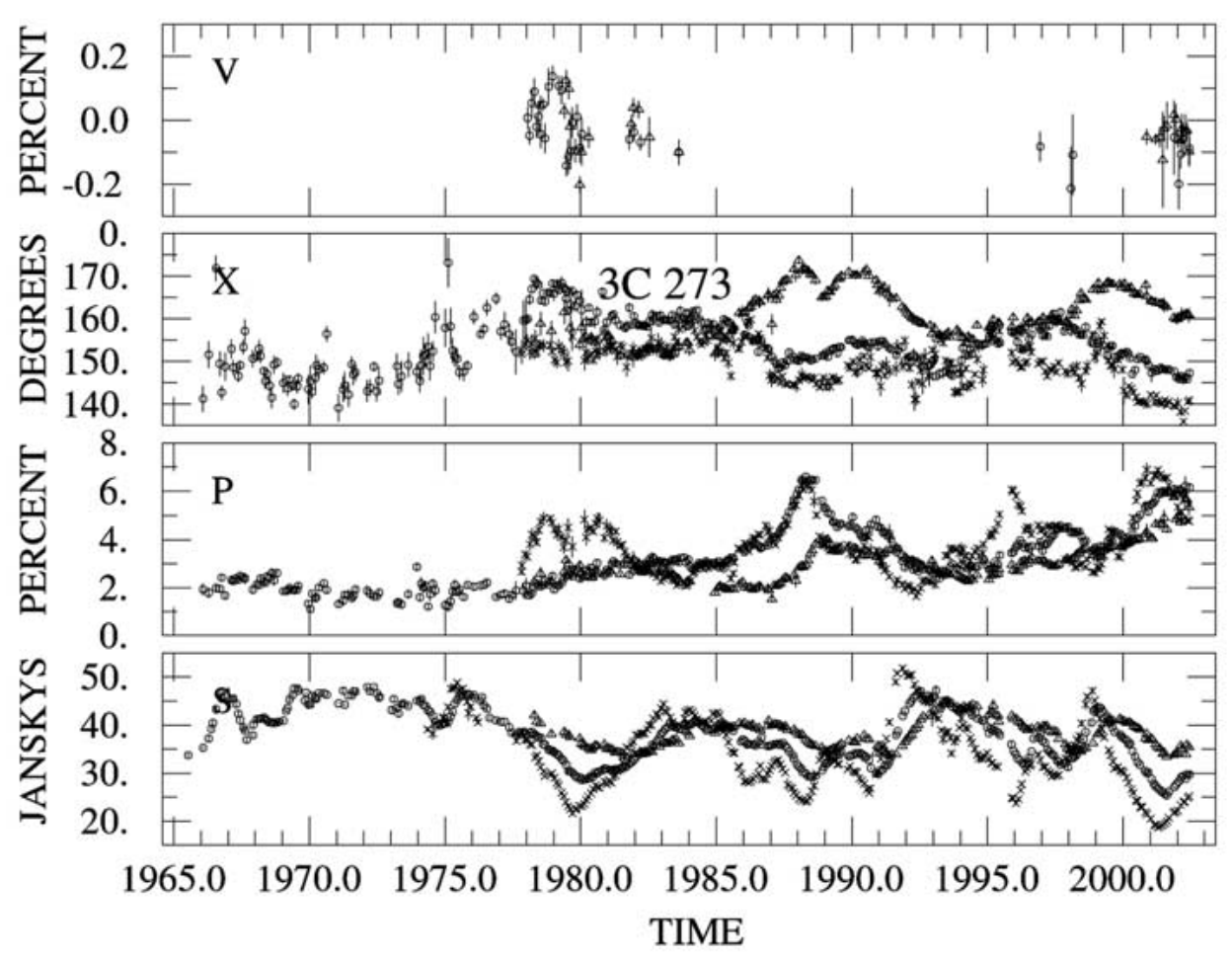

Figure 6. Monthly averages of the observed Stokes parameters for $3 \mathrm{C} 273$ at $4.8,8.0 \& 14.5 \mathrm{GHz}$, shown as $\triangle, O$, and $X$ respectively, versus time.

3C 84 This is one of two sources which was found to be time variable at $8.0 \mathrm{GHz}$ in the mid-1970s (Hodge and Aller, 1977). By 1978 the $8.0 \mathrm{GHz} \mathrm{cp}$ had changed sign (to positive $\mathrm{V}$ ), but it then underwent a steady decrease to negative values over the following four years (see Figure 3). An interesting point is that the 4.8 GHz observations (which were started in 1978) exhibited the same temporal change; there is no apparent difference in the cp at the two frequencies. In the recent data the $\mathrm{cp}$ is negative at $4.8 \mathrm{GHz}$ and not significantly different from zero at $8.0 \mathrm{GHz}$. The other striking feature about this Seyfert Galaxy is that while it has exhibited relatively strong $\mathrm{cp}$ it has remained essentially unpolarized in linear polarization (see Figure 5); this is a property it shares with the galactic center source (Bower, Falcke, and Backer, 1999; Bower et al., 2002; Sault and Macquart, 1999).

3C273 This is the second source that exhibited time variablilty at $8.0 \mathrm{GHz}$ during the mid-1970s; and it continued to show variability on time scales of less than a year in 1978-1979 (see Figure 3). V changed sign during this period at $8.0 \mathrm{GHz}$ (there was not enough $4.8 \mathrm{GHz}$ to clearly define the variability). The cp variability may be associated with the same event that produced the 
TABLE I

Average circular polarization

\begin{tabular}{lrrrrr}
\hline & & \multicolumn{2}{c}{$1978-1983$} & \multicolumn{2}{c}{$2001-2002$} \\
Source & $v(\mathrm{GHz})$ & $V(\%)$ & $\sigma_{V}(\%)$ & $V(\%)$ & $\sigma_{V}(\%)$ \\
\hline $0235+164$ & 4.8 & -0.241 & 0.127 & 0.017 & 0.021 \\
$(0235+164)$ & 8.0 & -0.124 & 0.099 & - & - \\
$3 \mathrm{C} 84$ & 4.8 & -0.066 & $0.032 \mathrm{~V}$ & -0.151 & $0.022 ?$ \\
$(0316+413)$ & 8.0 & -0.015 & $0.011 \mathrm{~V}$ & -0.017 & 0.030 \\
$3 \mathrm{C} 120$ & 4.8 & -0.012 & 0.136 & -0.019 & 0.057 \\
$(0430+052)$ & 8.0 & 0.033 & 0.045 & 0.223 & 0.375 \\
$0607-157$ & 4.8 & - & - & -0.227 & 0.038 \\
$(0607-157)$ & 8.0 & - & - & -0.199 & 0.116 \\
$4 \mathrm{C}-00.28$ & 4.8 & - & - & -0.511 & 0.077 \\
$(0743-006)$ & 8.0 & - & - & -0.288 & 0.174 \\
OJ 287 & 4.8 & -0.369 & 0.121 & - & - \\
$(0851+202)$ & 8.0 & -0.222 & 0.028 & - & - \\
$4 \mathrm{C} 39.25$ & 4.8 & 0.211 & 0.045 & - & - \\
$(0923+392)$ & 8.0 & -0.030 & 0.030 & - & - \\
$4 \mathrm{C} 49.22$ & 4.8 & - & - & -0.145 & 0.089 \\
$(1150+497)$ & 8.0 & - & - & - & - \\
$3 \mathrm{C} 273$ & 4.8 & -0.026 & $0.031 \mathrm{~V}$ & -0.029 & 0.023 \\
$(1226+023)$ & 8.0 & -0.005 & $0.012 \mathrm{~V}$ & -0.092 & 0.021 \\
$3 \mathrm{C} 279$ & 4.8 & 0.079 & $0.048 ?$ & -0.171 & 0.011 \\
$(1253-055)$ & 8.0 & -0.010 & $0.035 ?$ & 0.066 & 0.044 \\
$1510-089$ & 4.8 & - & - & - & - \\
$(1510-089)$ & 8.0 & 0.099 & 0.083 & - & - \\
$1519-273$ & 4.8 & - & - & -0.916 & 0.169 \\
$(1519-273)$ & 8.0 & - & - & -0.916 & 0.431 \\
$3 \mathrm{C} 345$ & 4.8 & 0.148 & $0.042 \mathrm{~V}$ & 0.095 & $0.025 ?$ \\
$(1641+399)$ & 8.0 & 0.014 & $0.029 \mathrm{~V}$ & 0.041 & 0.062 \\
$2134+004$ & 4.8 & - & - & 0.618 & 0.060 \\
$(2134+004)$ & 8.0 & - & - & - & - \\
$2145+067$ & 4.8 & - & - & -0.496 & 0.074 \\
$(2145+067)$ & 8.0 & - & - & - & - \\
BL LAC & 4.8 & 0.011 & 0.070 & - & - \\
$(2200+420)$ & 8.0 & -0.082 & 0.049 & - & - \\
\hline & & & & &
\end{tabular}




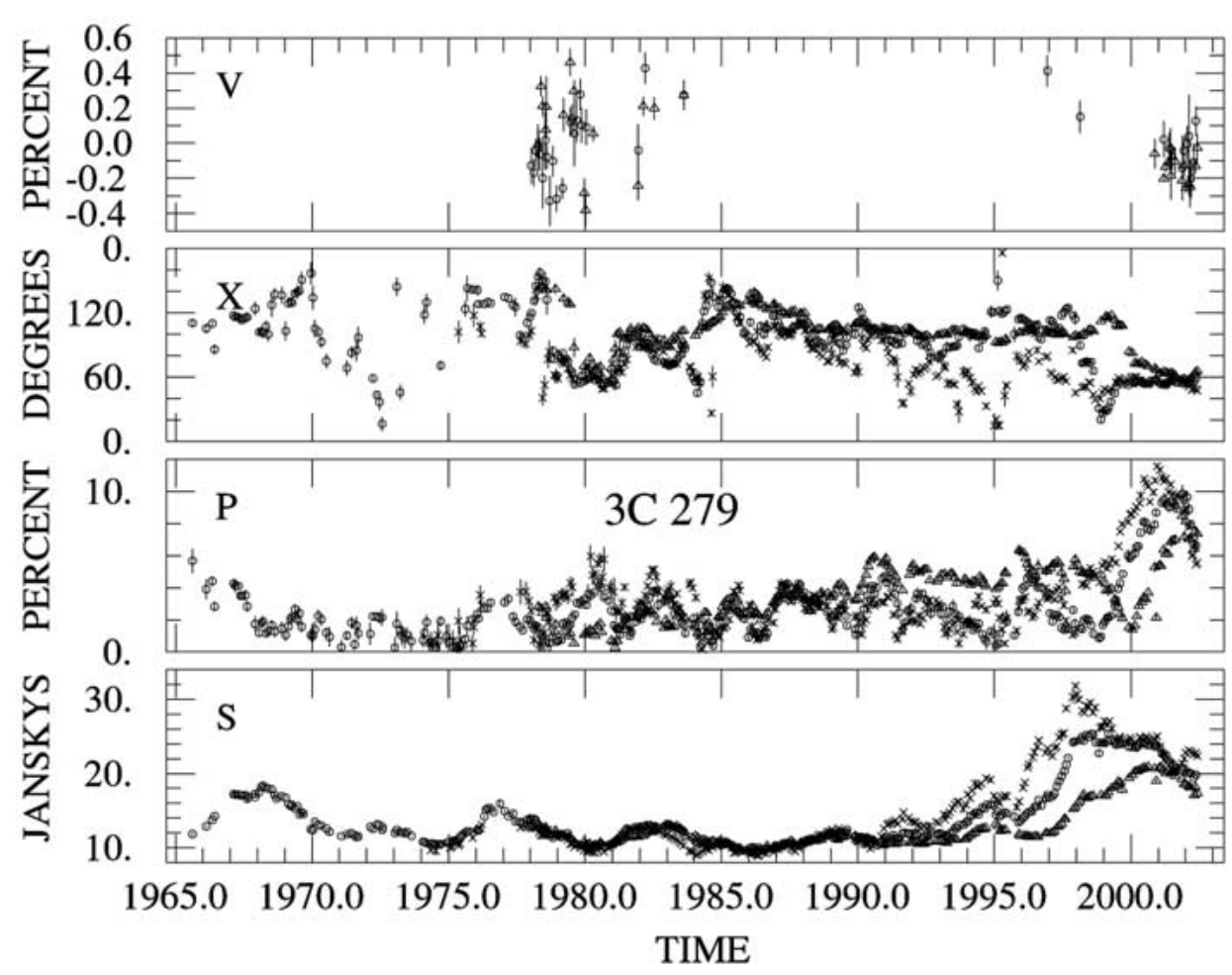

Figure 7. Monthly averages of the observed Stokes parameters for 3C 279 at $4.8,8.0 \& 14.5 \mathrm{GHz}$, shown as $\triangle, O$, and $X$ respectively, versus time.

rapid 2-cm linear polarization 'outburst', but there is insufficient long-term time coverage to rule out the possibility that this type of $\mathrm{cp}$ variability occurs all the time in the source. We have not detected significant cp during our more recent observations.

3C 279 The source was detected (positive V) in the the mid-1970s, exhibited apparently erratic variations during 1978-1979 (apparently coincident with rapid, large-amplitude changes in linear polarization) and recently exhibited negative $\mathrm{V}$ at $4.8 \mathrm{GHz}$ (see Figures 4 and 7). Homan and Wardle (1999) observed positive circular polarization in this source at $15 \mathrm{GHz}$ during 1996; it is not clear weather the aparent sign reversal (compared to our recent $4.8 \mathrm{GHz}$ results) is due to a temporal change in the source or to a spectral effect.

3C 345 This object, although essentially unpolarized in cp during the mid-1970s period, (Hodge and Aller, 1977), exibited relatively strong cp ( $\geq 0.5 \%)$ and variability on time scales of less than a year in 1978-1979 which corresponded to the onset of a large outburst in the source (see Figures 4 and 8). There was also an apparently rapid, large amplitude cp outburst in late 2001 (the $4.8 \mathrm{GHz}$ cp reached $0.4 \%$ ) on a time scale of a month or less (it is undersampled in 


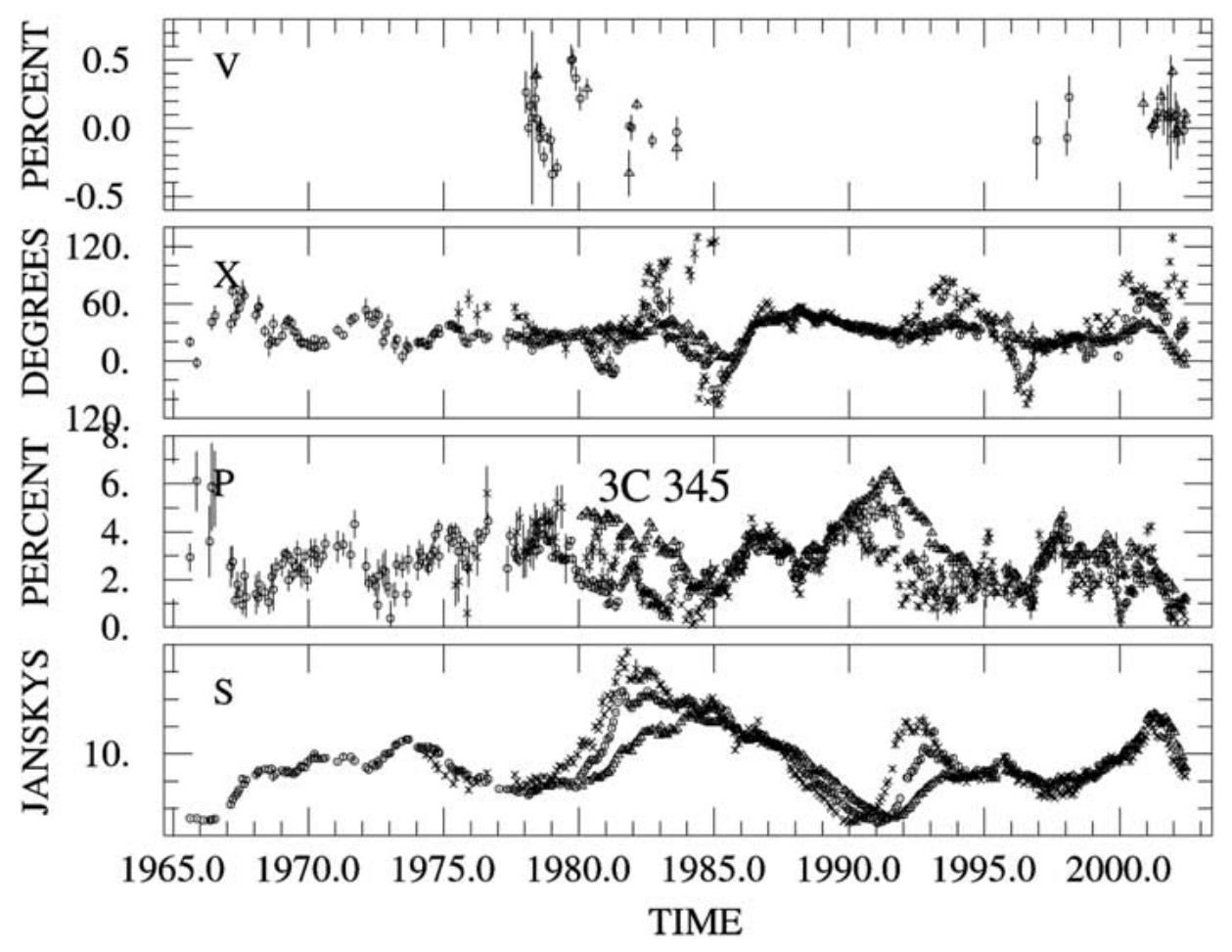

Figure 8. Monthly averages of the observed Stokes parameters for 3C 345 at $4.8,8.0$ \& $14.5 \mathrm{GHz}$, shown as $\triangle, O$, and $X$ respectively, versus time.

our observations); it associated with the onset of a new large outburst in the source, shich can be seen in Figure 8.

1519-273 We confirm the high cp (we found almost $1 \%$ ) at $4.8 \mathrm{GHz}$ reported in this BL Lac object (Macquart et al, 2000). The unusually high cp in this source appears to be explained by an intriging scintillation mechanism (Macquart and Melrose, 2000).

\section{Conclusions}

The results presented here and by others indicate that low levels of $\mathrm{cp}$ are common in active extragalactic sources. Many sources reach levels of $\geq 0.1 \%$, and some exceed a percent. Variations in cp occur on time scales ranging from weeks to decades; one object, believed to be an interday variable, has exhibited variations on time scales of days less (Macquart et al, 2000). The cp emission is often broadband in nature: we have observed simultaneous temporal variations at 4.8 and $8.0 \mathrm{GHz}$ in several sources. We have also observed rapid sign refersals in the CP. The strength 
of the cp does not appear to be correlated with the integrated linear polarization in a source, in fact one of the more strongly circularly polarized sources, $3 \mathrm{C} \mathrm{84}$, did not exhibit any detectable linear polarization for decades (it became weakly polarized at $14.5 \mathrm{GHz}$ after 2000, see Figure 5). However, 3C 273, 3C 279, and 3C 345 all exhibited $\mathrm{cp}$ when either the linear-polarization spectrum or the appearence of a new outburst indicate that a partially opaque component existed in the source. VLBA polarization maps have also shown that the cp appears to be emitted by components near or in the core of active sources, which are likely to be partially opaque (Homan and Wardle, 1999).

The behavior we have observed appears to be consistent with the hypothesis that the $\mathrm{cp}$ in these sources (not including the interday variable, PKS 1519-273) is produced by mode conversion in a relativistic magnitized plasma (Jones and O'Dell, 1977a; Jones and O'Dell, 1977b). The observed sign reversals with time in the observed $\mathrm{cp}$ of individual sources suggest that, if there are underlying fixed structures in the magnetic fields, they may be hidden by the the effects of the stocastic nature of mode conversion in a turbulent magnetic field structure. However the timescales and bandwidth of the $\mathrm{cp}$ variability, may still provide unique information concerning the physical scales of these turbulent structures.

\section{Acknowledgements}

The acqusition of the early measurements reported here was supported in part by the National Science Foundation under grants AST 76-00410, 78-24192, and 80-21250. The University of Michigan Radio Observatory is supported by the University of Michigan Department of Astronomy. HDA and MFA would like to acknowledge the hospitality of the MPIfR-Bonn where much of the analysis presented here was carried out.

\section{References}

Bower, G.C., Falcke, H. and Backer, D.C.: 1999, Detection of circular polarization in the galactic center black hole candidate Sagittarius A*, The Astrophysical Journal Letters 523, L29-L32.

Bower, G.C., Falcke, H., Sault, R.J. and Backer, D.C.: 2002, The spectrum and variability of circular polarization in Sagittarius A* from 1.4 to $15 \mathrm{GHz}$, The Astrophysical Journal 571, 843-855.

Hodge, P.E. and Aller, H.D.: 1977, Variations in the circular polarization of 3C 84 and 3C 273 at $8 \mathrm{GHz}$, The Astrophysical Journal 211, 669-674.

Homan, D.C. and Wardle, J.F.C.: 1999, Detection and measurement of parsec-scale circular polarization in four AGNS, The Astrophysical Journal 118, 1942-1962.

Homan, D.C., Attridge, J.C. and Wardle, J.F.C.: 2001, Parsec-scale circular polarization observations of 40 blazars, The Astrophysical Journal 556, 113-120.

Jones, T.W. and O'Dell, S.L.: 1997a, Transfer of polarized radiation in self-absorbed synchrotron sources. I. Results for a homogeneous source, The Astrophysical Journal 214, 522-539. 
Jones, T.W. and O'Dell, S.L.: 1977b, Transfer of polarized radiation in self-absorbed synchrotron sources. II. Treatment of inhomogeneous media and calculation of emergent polarization, The Astrophysical Journal 215, 236-246.

Komesaroff, M.M., Roberts, J.A., Milne, D.K., Rayner, P.T. and Cooke, D.T.: 1984, Circular and linear polarization variations of compact radio sources, MNRAS 208, 409-425.

Legg, M.P.C. and Westfold, K.C.: 1968, Elliptical polarization of synchrotron radiation, The Astrophysical Journal 154, 499-514.

Macquart, J.-P., Kedziora-Cudczer, L., Rayner, D.P. and Jauncey, D.L.: 2000, Strong, variable circular polarization in PKS 1519-273, The Astrophysical Journal 538, 623-627.

Macquart, J.-P. and Melrose, D.B.: 2000, Scintillation-induced circular polarization in pulsars and quasars, The Astrophysical Journal 545, 798-806.

Rayner, D.P., Norris, R.P. and Sault, R.J.: 2000, Radio circular polarization of active galaxies, MNRAS 319, 484-496.

Sault, R.J. and Macquart, J.-P.: 1999, Confirmation and analysis of circular polarization from Sagittarius A*, The Astrophysical Journal Letters 526, L85-L88.

Wardle, J.F.C., Homan, D.C., Ojha, R. and Roberts, D.H.: 1998, Electron-positron jets associated with the quasar 3C 279, Nature 395, 457-461.

Weiler, K.W. and de Pater, I.: 1983, A catalogue of high accuracy circular polarization measurements, The Astrophysical Journal Supplement 52, 293-327. 